\title{
Clinical and laboratory status of patients with chronic Chagas disease living in a vector-controlled area in Minas Gerais, Brazil, before and nine years after aetiological treatment
}

\author{
Marta de Lana ${ }^{1 /+}$, Leonardo A Lopes², Helen R Martins², Maria T Bahia ${ }^{3}$, \\ Girley F Machado-de-Assis ${ }^{2}$, Ana P Wendling ${ }^{4}$, Olindo A Martins-Filho ${ }^{4}$, Roberto A Montoya ${ }^{5}$, \\ João CP Dias ${ }^{4}$, Pedro Albajar-Viñas ${ }^{6}$, José R Coura ${ }^{6}$
}

\begin{abstract}
'Departamento de Análises Clínicas, Escola de Farmácia ${ }^{2}$ Núcleo de Pesquisas em Ciências Biológicas³Departamento de Ciências Biológicas, Instituto de Ciências Exatas e Biológicas, Universidade Federal de Ouro Preto, Rua Costa Sena 175, 35400-000 Ouro Preto, MG, Brasil ${ }^{4}$ Instituto René Rachou-Fiocruz, Belo Horizonte, MG, Brasil ${ }^{5}$ Organização Pan-Americana da Saúde, Brasília, GO, Brasil ${ }^{6}$ Laboratório de Doenças Parasitárias, Instituto Oswaldo Cruz-Fiocruz, Rio de Janeiro, RJ, Brasil
\end{abstract}

Twenty-eight Chagas disease patients (CD), 22 with the indeterminate clinical form (IND) and six with the cardiac or digestive form (CARD/DIG), were treated with benznidazole and underwent clinical and laboratorial analysis before (IND and CARD/DIG) and nine years after [patients after treatment (CDt), patients with the indeterminate clinical form at treatment onset (INDt) and with the cardiac or digestive form at treatment onset (CARD/DIGt)] treatment. The data demonstrate that $82.1 \%$ of CDt patients (23/28) remained clinically stable and 95.4\% of the INDt (21/22) and 33.3\% of the CARD/DIGt (2/6) patients showed unaltered physical and laboratorial examinations. The clinical evolution rate was $2 \% / y e a r$ and was especially low in INDt patients $(0.5 \% / y e a r)$ relative to CARD/DIGt patients (7.4\%/year). Positive haemoculture in treated patients was observed in $7.1 \%$ of the cases. None of the INDt (0/21) and $33.3 \%$ of the CARD/DIGt (2/6) patients displayed positive cultures. The PCR presented a positive rate significantly higher $(85.2 \%, 23 / 27)$ than haemoculture and two samples from the same patient revealed the same result $57.7 \%$ of the patients. Conventional serology-ELISA on 16 paired samples remained positive in all individuals. Semi-quantitative ELISA highlighted significant decreases in reactivity, particularly in INDt relative to IND. Non-conventional serology-FC-ALTA-IgG, after treatment, showed positive results in all sera and 22 paired samples examined at seven and nine years after treatment, demonstrated significantly lower reactivity, particularly in INDt patients. This study was retrospective in nature, had a low number of samples and lacked an intrinsic control group, but the data corroborate other results found in the literature. The data also demonstrate that, even though a cure has not been detected in the none-treated patients, the benefits for clinical evolution were selectively observed in the group of INDt patients and did not occur for CARD/DIGt patients.

Key words: Chagas disease - aetiological treatment - benznidazole - clinical-laboratorial evaluation - clinical evolution

Chagas disease has been brought under control in several Latin American countries due to successive vector control programs along with successful blood transfusion control (WHO 2005). Nevertheless, the large numbers of individuals infected with Trypanosoma cruzi still represent a serious public health problem and an important challenge for physicians and the scientific community. Therefore, continuing epidemiological surveillance activities relating to Chagas disease are recommended (Dias 2002).

Despite the urgent need to develop drugs that are more effective for aetiological treatment of Chagas disease, the only compounds currently available are

Financial support: FAPEMIG (PPSUS/05, 3242/06), CNPq (481097/2004-0), SESU/MEC, PROEX/UFOP

+ Corresponding author: delana@nupeb.ufop.br

Received 13 February 2009

Accepted 9 October 2009 benznidazole and nifurtimox (Coura \& Castro 2002). The general consensus is that there is greater therapeutic effectiveness during the acute phase of the disease (70-90\% of cure), in recent infections (present for less than 12 years) and among young patients compared with individuals treated during late chronic infection (Cançado 1997, Anonymous 2005).

In addition to the lower efficacy of chemotherapy applied to chronic infection, the belief that autoimmune events play an important underlying role in Chagas pathogenesis, even after the post-therapeutic parasite clearance (Coura et al. 1997), has led several investigators to conclude that it is not worth treating chronic Chagas disease patients (CD). However, a few follow-up studies on treated CD have pointed out that, in fact, despite the autoimmune mechanisms triggered by $T$. cruzi infection, most tissue damage is indeed dependent on the presence of the parasite (Viotti et al. 1994). In fact, several authors have also demonstrated that real benefits are observed following specific chemotherapy, regardless of whether a parasitological cure is achieved (Segura et. al. 1994, Viotti et al. 1994, 2006, Galerrano \& Sosa 2000, Suasnábar et al. 2000, Streiger et al. 2004, Fabbro et al. 2007). These ef- 
fects have been observed especially when specific treatment was offered to young patients or to those with mild disease, such that better prognosis for the infection or even regression of the lesions or clinical cardiac or digestive symptoms was observed (Fabbro et al. 2007). Similar findings have also been observed following treatment of experimental T. cruzi infections (Andrade et al. 1991).

However, controversy still exists regarding the real effects of these drugs on the clinical evolution of Chagas disease, especially during the chronic phase of the disease (Macedo \& Silveira 1987, Braga et al. 2000, Lauria-Pires 2000). The controversy is most likely due to the use of different treatment schemes and/or evaluation protocols (Bahia et al. 2006). Consequently, despite the evidence that trypanocidal therapy may impact positively on the clinical course of the disease in chronic patients (Sosa \& Segura et al. 2006, Bern et al. 2008), there is neither a consensus nor sufficient data to support the routine use of the aetiologic treatment of this condition (Marin-Neto et al. 2009). In fact, the major concern to be considered with regard to truly demonstrating the efficacy of treatment and the beneficial impact of aetiological treatment on the clinical evolution of Chagas disease is the need for long-term follow-up of treated patients (Coura \& Castro 2002). In this context, a recent randomised doubleblind controlled clinical trial (BENEFIT) was launched in countries with a high incidence of Chagas disease to clarify the role of trypanocidal therapy in preventing cardiac disease progression and death using the standardised protocol for treatment, clinical evaluation and cure assessment (Marin-Neto et al. 2009).

Extending the work on this issue, the intention of the present investigation was to perform a comparative evaluation of $\mathrm{CD}$ in a vector-controlled area [the municipality of Berilo, Minas Gerais (MG), Brazil] before and nine years after aetiological treatment. This study covered important aspects of post-therapeutic cure criteria, including the parasitological/serological status and clinical scores of treated CD.

\section{PATIENTS AND METHODS}

Patient sample - Twenty-eight individuals, 6-37 years old (9 males and 19 females), were evaluated. All of the subjects were living in the Jequitinhonha Valley, a vector-controlled area within the municipality of Berilo, MG, Brazil (Fig. 1). The major demographic, clinical and laboratory characteristics of all the subjects, obtained before and nine years after treatment with benznidazole, are summarised in Table I.

Clinical and laboratory evaluation - Before treatment, the laboratory diagnosis of Chagas disease was confirmed by two serological tests [indirect immunofluorescence and indirect haemagglutination (IHA)] (data not shown). Additional serological analysis was performed using conventional methods.

All of the CD were physically and clinically examined and electrocardiograms and chest X-rays were recorded. Cases of cardiopathy were classified according to clinical and electrocardiographic criteria as recommended by OMS/OPAS (1974). Twenty-two out of the 28 patients

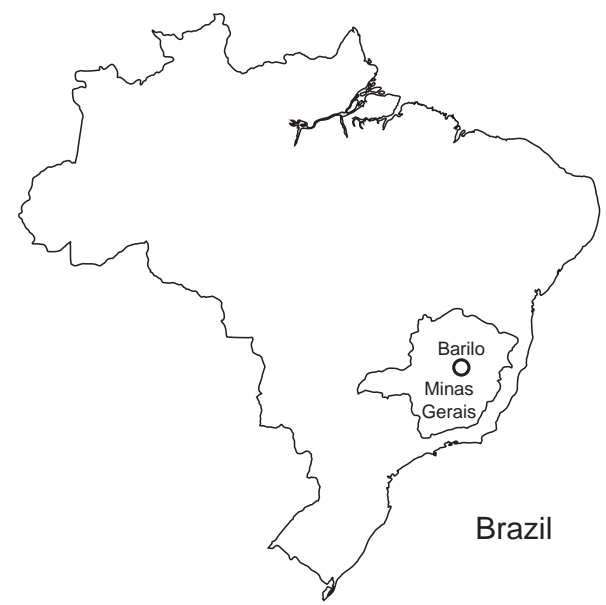

Fig. 1: study area. The municipality of Berilo is located in the Jequitinhonha Valley (10 $57^{\prime} 06^{\prime \prime}$; $\left.42^{\circ} 27^{\prime} 56^{\prime \prime} \mathrm{W}\right)$, in the northwest of the state of Minas Gerais, which has been reported to be one of the most important endemic areas for Chagas disease in Brazil. This municipality is at an altitude of $401 \mathrm{~m}$, covering an area of $581.5 \mathrm{~km}^{2}$ and has 13,214 inhabitants, according to the 2007 IBGE census, who mostly live in the rural zone. The economy is based on agriculture and cattleranching activities [ibge.cidades@; available from: http://www.ibge. gov.br/cidadesat (search for Berilo)].

presented the indeterminate clinical form of the disease, while five were classified as mild cardiac patients and one as a megaoesophagus group I digestive patient, in accordance with Resende et al. (1960). The patients were observed for any abnormal renal or hepatic conditions and they all received specific treatment with benznidazole.

The treatment consisted of administration of the nitroderivative benznidazole (N-benzyl-2-nitroimidazole acetamide - Roche ${ }^{\circledR}$ ). The therapeutic scheme consisted of $5-10 \mathrm{mg} / \mathrm{kg} /$ day for $40-60$ consecutive days. During treatment, all patients were re-evaluated at regular intervals of 15 days or whenever any side effects were observed. The most frequent side effects observed were anorexia, muscle pain and exanthema. One case of severe adverse effects was documented and this individual's treatment was temporarily interrupted until the laboratorial parameters returned to normal.

After treatment, the laboratory evaluation consisted of parasitological and serological tests, as follows:

Parasitological tests - For parasitological tests, two samples from each patient (collected within a one-year interval) were employed. Blood culturing was performed using a volume of $30 \mathrm{~mL}$ of blood, processed as described by Chiari et al. (1989). Blood samples for PCR were collected in an equal volume of guanidine $6 \mathrm{M}$ and EDTA $0.2 \mathrm{M}, \mathrm{pH}$ 8.0. The DNA extraction and PCR reaction were performed in accordance with Gomes et al. (1998). Positive, negative and reagent controls were processed in parallel in each assay in all stages. The first sample was subjected to two independent PCR analyses. Once the results were reproducible among the two different extractions in the first sample, the second sample was 
submitted to a single PCR analysis. The primers S35 and S36 were used for amplification of kDNA minicircles in the presence of Taq DNA polymerase (Platinum, Invitrogen). The products were verified on polyacrylamide gels and the PCR results were compiled after two tests, with the results reported as negative or positive.

Serological tests - Conventional serological evaluations were performed by means of ELISA and IHA tests and they confirmed the diagnosis of Chagas disease for all the patients (data not shown). Additionally, conventional ELISA was performed on 16 samples paired with those stored at the Laboratório de Doenças Parasitárias (Instituto Oswaldo Cruz-Fiocruz, Rio de Janeiro, RJ, Brazil), with the aim of investigating whether there was any decrease in antibody levels after treatment. ELISA was performed essentially as described by Voller et al. (1976) but modified by using $4.5 \mu \mathrm{g} / \mathrm{mL}$ antigen, a serum dilution of 1:80 and peroxidase-conjugated anti-human IgG conjugate. The results are expressed as optical densities (OD) and as "changes in IgG reactivity" (i.e., ODt9-ODt0). Furthermore, semiquantitative ELISA was applied to characterise the changes in serological reactivity following aetiological treatment. The semiquantitative ELISA was first established using serial dilutions (1:80; $1: 160 ; 1: 320 ; 1: 640 ; 1: 1,280$ and $1: 2,560)$ of pooled serum samples from infected non-treated patients (INT), treated and not cured patients (TNC), treated and cured patients (TC) and non-infected individuals (NI) that were stored at the Laboratório de Doença de Chagas, Instituto René Rachou-Fiocruz, Belo Horizonte, MG, Brazil.

Non-conventional serological testing was performed by means of analysing anti-live-trypomastigote antibodies (FC-ALTA-IgG) in sera from all 28 patients (collected 9 years after aetiological treatment), as described by Cordeiro et al. (2001). The test was considered positive when the percentage of positive fluorescent parasites (PPFP) was greater than $20 \%$. Additionally, FC-ALTAIgG was performed on 22 samples paired with those collected seven years after treatment, which were stored at the Laboratório de Doença de Chagas, Núcleo de Pesquisas em Ciências Biológicas, Universidade Federal de Ouro Preto, Ouro Preto, MG, Brazil.

The clinical and physical examinations and the clinical classifications of the patients were performed by our clinical team following the same criteria that had been used prior to treatment.

Statistical analysis - Comparative analyses of serological status before and after treatment with benznidazole, including the IgG level detected by conventional ELISA, semiquantitative ELISA and FC-ALTA-IgG, were performed using the Wilcoxon test. The results were considered significant when $\mathrm{p}<0.05$. For comparative analysis, three groups of subjects were used: CD, patients with the indeterminate clinical form of Chagas disease (IND) and patients with cardiac/digestive clinical manifestations of Chagas disease (CARD/DIG), evaluated before (CD, IND and CARD/DIG) and nine years after [CD after treatment (CDt), patients with the indeterminate clinical form of Chagas disease at treatment onset (INDt) and patients with the cardiac or diges- tive form of Chagas disease at treatment onset (CARD/ DIGt)] treatment with benznidazole.

Ethics - This study complied with resolution 196/1996 from the National Health Council for research involving humans and all of the patients and legal guardians read and signed the consent form (Committee on Human Experimentation from $\mathrm{CPqRR}$, Fiocruz, Belo Horizonte, MG - process 007/2002).

\section{RESULTS}

Clinical evaluation after aetiological treatment - The comparative analysis of the clinical status of the $28 \mathrm{CD}$ before and after aetiological treatment with benznidazole is summarised in Table I and Fig. 2. Although the number of patients evaluated was low, five out of the 28 patients (17.9\%) had progressive disease (Fig. 2A), reaching a clinical evolution rate of $2 \%$ nine years after treatment (Fig. 2B).

Disease progression was observed in only one out of the 22 patients (4.6\%) who had presented the indeterminate clinical form before treatment (Fig. 2A). This individual's case evolved to a mild cardiac clinical form (patient 27) (Table I), leading to a clinical evolution rate of $0.5 \% /$ year (Fig. 2B).

On the other hand, four out of the six patients (66.7\%) who displayed clinical manifestations of Chagas disease (CARD/DIG) before treatment (Fig. 2A) evolved towards a more severe clinical status (patients 4, 8, 15 and 24) (Fig. 2A), resulting in a clinical evolution rate of 7.4\%/year (Fig. 2B). Fig. 2C illustrates the expected natural clinical evolution rate for untreated Chagas disease according to previous reports (Viotti et al. 1994, 2006, Gallerano \& Sosa 2000, Dias 2006, Fabbro et al. 2007).

Parasitological analysis - Blood culturing was performed after treatment for all 28 of the treated CD (two blood samples from each patient). Two out of the 28 treated patients (7.1\%) showed positive blood cultures: both of them presented clinical manifestations of Chagas disease before treatment (patients 4 and 9) (Table I). None of the patients who displayed the indeterminate clinical form of Chagas disease before treatment showed positive blood cultures nine years after treatment, whereas 33.3\% (2/6) of the patients with clinical manifestations before treatment (CARD/DIG) presented positive blood cultures.

PCR analysis demonstrated that four out of the 27 CD (14.8\%) displayed negative PCR results nine years after aetiological treatment (patients 10, 16, 18 and 22) after evaluation of two different samples, while $85.2 \%$ (23/27) showed positive results (Table I). The percentages of agreement between the results of two distinct samples were $57.7 \%$, with $69.2 \%$ positive in the first sample and $59.3 \%$ in the second. The percentages of indeterminate patients displaying negative or positive PCR were 20\% (4/20) and $80 \%(16 / 20)$, respectively, whereas for patients with clinical manifestations before treatment, these frequencies were $0 \%(0 / 6)$ and $100 \%(6 / 6)$, respectively (Table I).

Conventional and semiquantitative ELISA - The serological analyses performed by conventional ELISA on 16 paired serum samples are shown in Fig. 3. Interest- 
TABLE I

Demographic, clinical and laboratorial features of Chagas disease patients from municipality of Berilo, Jequitinhonha Valley, Minas Gerais, Brazil, before and after treatment with benznidazole

\begin{tabular}{|c|c|c|c|c|c|c|c|}
\hline \multirow[b]{2}{*}{ Patient } & \multicolumn{3}{|c|}{ Before treatment } & \multicolumn{4}{|c|}{ After treatment } \\
\hline & $\begin{array}{c}\text { Age } \\
\text { (years) }\end{array}$ & Sex & $\begin{array}{l}\text { Clinical } \\
\text { form }\end{array}$ & $\begin{array}{l}\text { Clinical } \\
\text { form }\end{array}$ & $\mathrm{HC}$ & $\begin{array}{c}\text { PCR } \\
\text { (1st/2nd sample) }\end{array}$ & $\begin{array}{l}\text { FC-ALTA- } \\
\text { IgG t9 }{ }^{a}\end{array}$ \\
\hline 1 & 6 & M & IND & IND & neg & neg/pos & 51.0 \\
\hline 2 & 10 & $\mathrm{~F}$ & IND & IND & neg & neg /pos & 88.1 \\
\hline 3 & 11 & M & IND & IND & neg & - & 86.6 \\
\hline 4 & 14 & $\mathrm{~F}$ & CARD discrete & CARD moderate & pos & pos/pos & 92.9 \\
\hline 5 & 14 & F & IND & IND & neg & pos/pos & 74.2 \\
\hline 6 & 16 & F & IND & IND & neg & pos/neg & 75.4 \\
\hline 7 & 21 & $\mathrm{~F}$ & IND & IND & neg & pos/pos & 85.3 \\
\hline 8 & 22 & $\mathrm{~F}$ & CARD discrete & CARD severe & neg & pos/neg & 93.0 \\
\hline 9 & 23 & F & CARD discrete & CARD discrete & pos & pos/neg & 81.8 \\
\hline 10 & 24 & F & IND & IND & neg & neg/neg & 96.1 \\
\hline 11 & 24 & F & IND & IND & neg & pos/neg & 94.0 \\
\hline 12 & 25 & F & IND & IND & neg & pos/pos & 82.6 \\
\hline 13 & 25 & $\mathrm{~F}$ & NI & IND & neg & pos/neg & 96.5 \\
\hline 14 & 26 & $\mathrm{~F}$ & IND & IND & neg & pos/pos & 95.1 \\
\hline 15 & 26 & F & DIG grade I & DIG grade III & neg & pos/neg & 87.8 \\
\hline 16 & 28 & $\mathrm{~F}$ & IND & IND & neg & neg/neg & 90.5 \\
\hline 17 & 29 & $\mathrm{~F}$ & IND & IND & neg & pos/neg & 87.3 \\
\hline 18 & 29 & M & IND & IND & neg & neg/neg & 91.3 \\
\hline 19 & 30 & M & IND & IND & neg & neg/pos & 88.3 \\
\hline 20 & 30 & M & NI & IND & neg & neg/pos & 59.0 \\
\hline 21 & 30 & M & CARD discrete & CARD discrete & neg & neg/pos & 93.6 \\
\hline 22 & 31 & M & NI & IND & neg & neg/neg & 90.8 \\
\hline 23 & 31 & M & IND & IND & neg & pos/pos & 91.1 \\
\hline 24 & 33 & M & CARD discrete & CARD severe & neg & pos/pos & 82.4 \\
\hline 25 & 33 & M & IND & IND & neg & pos/pos & 91.5 \\
\hline 26 & 34 & M & IND & IND & neg & pos/pos & 85.6 \\
\hline 27 & 37 & $\mathrm{~F}$ & IND & CARD discrete & neg & pos/pos & 90.4 \\
\hline 28 & 37 & $\mathrm{~F}$ & IND & IND & neg & pos/neg & 41.9 \\
\hline
\end{tabular}

$a$ : results expressed in percentage of positive fluorescent parasites; CARD: cardiac clinical form; DIG: digestive clinical form; HC: haemoculture; IND: indeterminate clinical form; neg: negative result; NI: not informed; pos: positive results; -: not performed.

ingly, although positive results from ELISA were observed in all 16 samples tested, a significant decrease in IgG reactivity was detected after treatment, selectively in the group of IND patients (Fig. 3A, B).

Four serum samples (patients 1, 11, 17 and 20) presented larger decreases in IgG reactivity (Table II) and all of them had the indeterminate clinical form of the disease (Table I). Smaller decreases in IgG reactivity were observed in three serum samples (patients 9, 15 and 21) (Table II) and all of them presented clinical manifestations of Chagas disease before treatment (Table I).

The semiquantitative ELISA was first established by assaying pooled serum samples from INT, TNC and TC $\mathrm{CD}$, along with samples from NI. The aims were to standardise the methodology and establish the interpretation criteria for evaluating post-therapeutic cure (Fig. 4, top graph). Data analysis demonstrated that IgG reactivity from 1:80 up to 1:640 was able to discriminate between Chagas disease samples (INT and TNC) and non-CD (TC and NI) (Fig. 4, top graph). This approach was used to analyse the mean IgG reactivity from 16 paired serum samples obtained before and nine years after treatment. The results demonstrated changes in $\mathrm{CD}$ vs. $\mathrm{CDt}$ and IND vs. INDt, which were similar to the profile observed for INT vs. TNC. On the other hand, no changes in mean IgG reactivity were observed for CARD/DIG vs. CARD/DIGt (Fig. 4, middle graph). The analysis of IgG reactivity in individual samples at selected serum dilutions (1:80-1:640) demonstrated that 1:320 was the most promising serum dilution, capable of identifying all of the CD as positive before treatment (IND and CARD/ DIG) and capable of discriminating between serological 


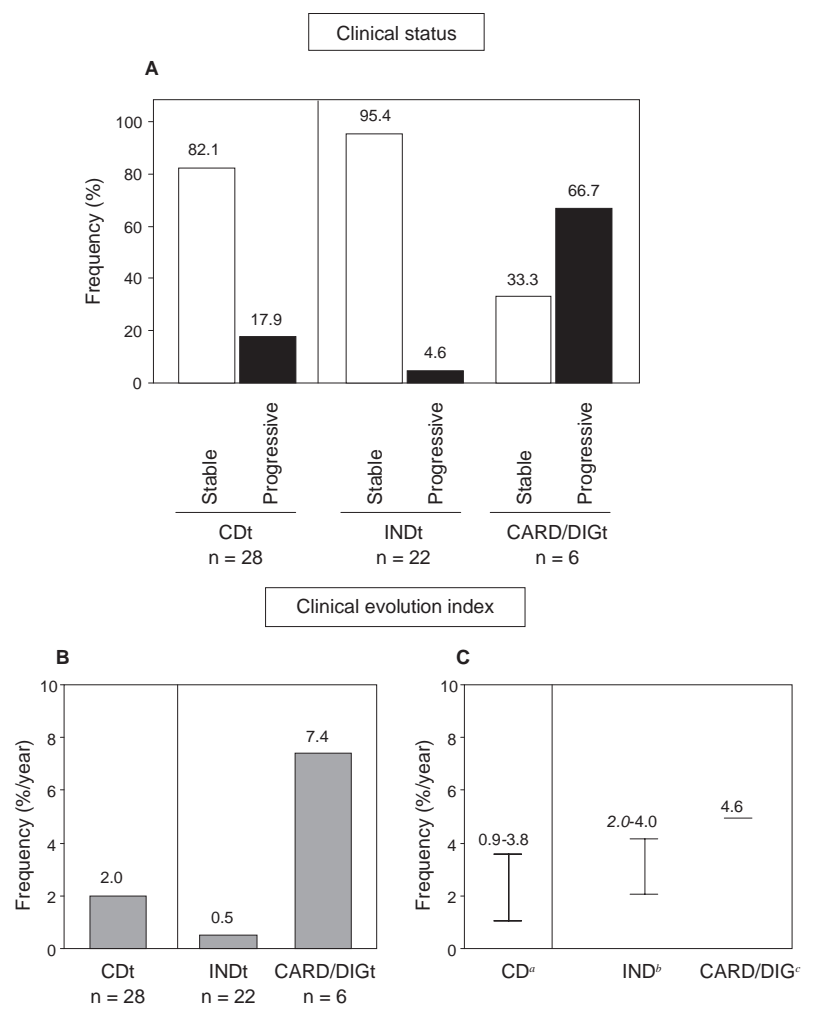

Fig. 2: clinical evolution characteristics of Chagas disease patients categorized as Chagas disease (CDt) and further categorized as indeterminate (INDt) or symptomatic (CARD/DIGt), based on their clinical scores at the start of the study. Clinical status (A) and clinical evolution rates (B) of CDt, INDt and CARD/DIGt individuals, nine years after etiological treatment with benznidazole. Expected clinical evolution rates $(C)$ of untreated CD, IND and CARD/DIG individuals, according to previous reports. $a$ : Viotti et al. (1994), Gallerano \& Sosa (2000), Fabbro et al. (2007); b: Dias (2006); c: Fabbro et al. (2007).

reactivities after treatment, particularly when comparing IND vs. INDt (Fig. 4, bottom graph). Specifically, at serum dilution 1:320, three serum samples (patients 1, 17 and 20) that all presented IND displayed IgG reactivity that was lower than the cut-off. No significant decrease in IgG reactivity was observed in the patients with clinical manifestations of Chagas disease (CARD/DIG vs. CARD/DIGt) (Fig. 4, bottom graph).

Non-conventional anti-live trypomastigote antibody analysis - FC-ALTA-IgG - The serological analyses performed using non-conventional FC-ALTA-IgG on 22 paired serum samples are shown in Fig. 5. Although all 22 of the samples tested were positive using FC-ALTAIgG, a significant decrease in mean IgG reactivity was detected after treatment, selectively in the group of IND patients (Fig. 5). A change in individual IgG reactivity was observed selectively in INDt (INDt7 vs. INDt9), with three serum samples (patients 1, 20 and 28) displaying the lowest PPFP values (Fig. 5).

\section{DISCUSSION}

One of the major challenges in investigating Chagas disease is the complexity of evaluating the impact of che-
TABLE II

Changes of seric IgG reactivity in Chagas disease patients from municipality of Berilo, Jequitinhonha Valley, Minas Gerais, Brazil, before and after treatment with benznidazole

\begin{tabular}{lccc}
\hline & Before treatment & After treatment & Change of reactivity \\
\cline { 2 - 4 } Patient & ELISA t0 & ELISA t9 & (ODt9-ODt0) \\
\hline $1^{a}$ & 0.71 & 0.33 & 0.38 \\
$9^{b}$ & 0.73 & 0.69 & 0.04 \\
$11^{a}$ & 0.77 & 0.55 & 0.22 \\
12 & 0.49 & 0.49 & 0 \\
13 & 0.67 & 0.58 & 0.09 \\
$15^{b}$ & 0.51 & 0.47 & 0.04 \\
16 & 0.66 & 0.59 & 0.07 \\
$17^{a}$ & 0.57 & 0.36 & 0.21 \\
18 & 0.61 & 0.55 & 0.06 \\
$20^{a}$ & 0.36 & 0.21 & 0.15 \\
$21^{b}$ & 0.64 & 0.63 & 0.01 \\
22 & 0.72 & 0.58 & 0.14 \\
23 & 0.58 & 0.58 & 0 \\
26 & 0.73 & 0.66 & 0.07 \\
27 & 0.79 & 0.71 & 0.08 \\
28 & 0.59 & 0.43 & 0.16 \\
\hline
\end{tabular}

ELISA results expressed in optical density (0D). $a$ : patients with higher changes in IgG reactivity; $b$ : patients with lower changes in IgG reactivity; t0: sample collected before treatment; t9: sample collected after treatment.
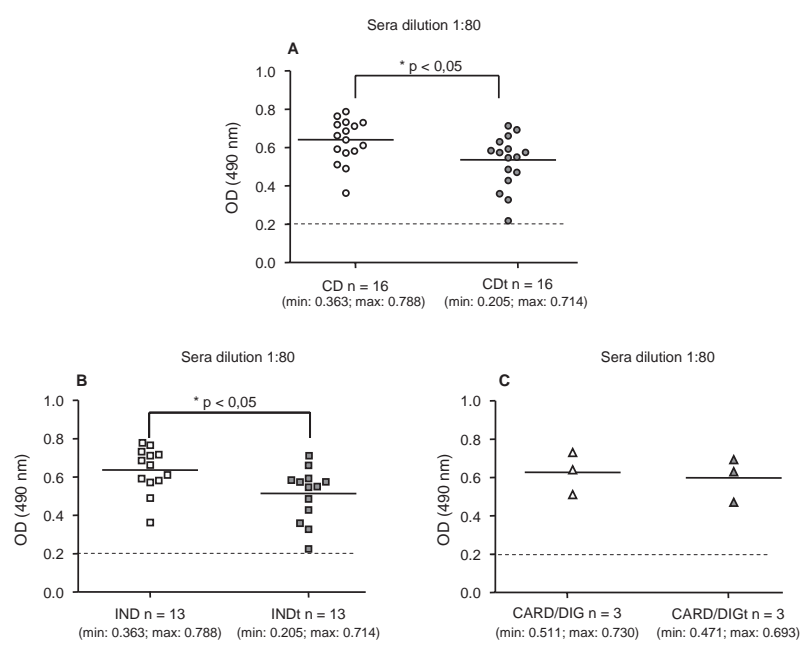

Fig. 3: serological reactivity of anti-Trypanosoma cruzi antibodies detected by conventional ELISA in Chagas disease patients (CD), categorized as indeterminate (IND) or symptomatic (CARD/DIG) based on their clinical scores at the start of treatment. The data are reported as the optical density (OD) of IgG reactivity at serum dilution 1:80, assessed before (CD, IND and CARD/DIG) and nine years after (CDt, INDt, CARD/DIGt) etiological treatment with benznidazole. Comparative analyses between the pairs of clinical groups were performed using the nonparametric Wilcoxon test and significant differences at $\mathrm{p}<0.05$ are highlighted with an asterisk. Minimum and maximum OD values for each clinical group are provided in this figure. 
motherapy on the clinical and laboratory characteristics of treated patients. Controversy still exists in relation to data that show that aetiological treatment has a positive impact and leads to diminished clinical evolution, mainly due to the different approaches and methods used by different authors (Bahia et al. 2006). This situation is exacerbated when managing treated chronic CD, since a longer follow-up investigation becomes necessary. Additionally, despite the lack of consensual cure criteria, a large number of laboratory methodologies (Coura

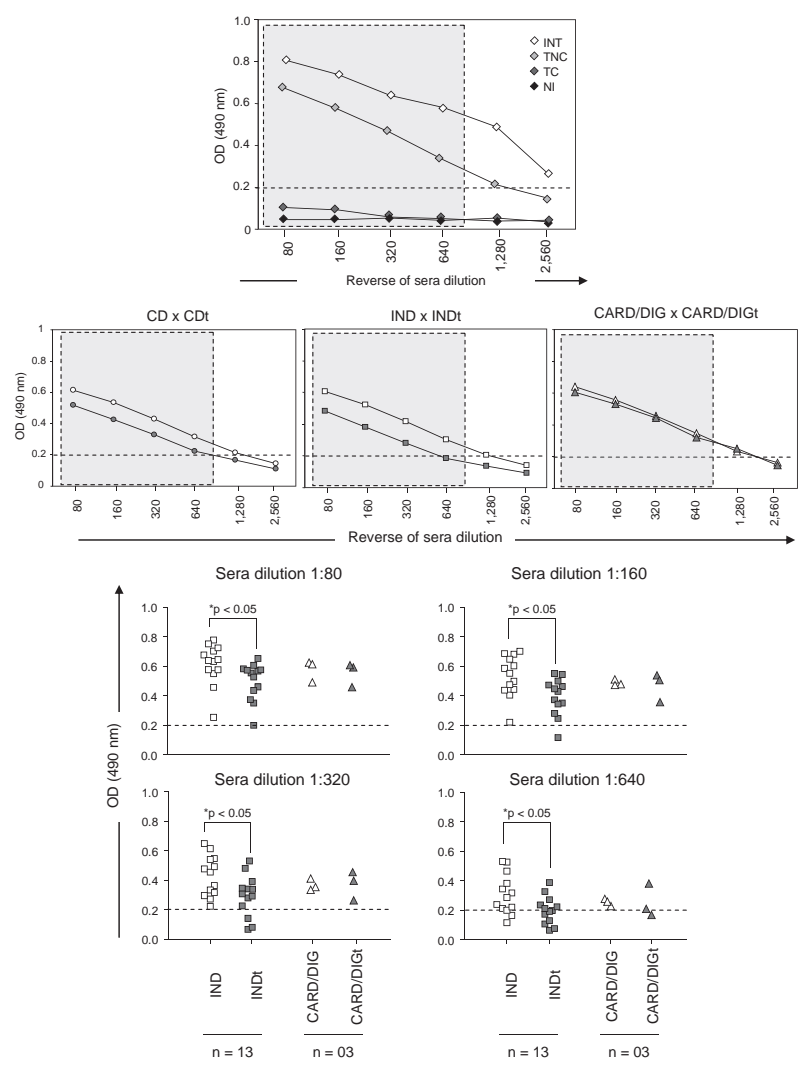

Fig. 4: serological reactivity of anti-Trypanosoma cruzi antibodies detected by semiquantitative ELISA in Chagas disease patients (CD), categorized as indeterminate (IND) or symptomatic (CARD/DIG) based on their clinical scores at the start of treatment. Semiquantitative ELISA was first established by assaying pooled serum samples from infected and not treated (INT), treated but not cured (TNC), treated and cured (TC) Chagas disease patients and from not infected individuals (NI), at serial serum dilutions (1:80, 1:160, 1:320, 1:640, 1:1280 and 1:2560). The IgG reactivity from 1:80 up to 1:640 was able to discriminate between Chagas disease samples (INT and TNC) and non-Chagas disease patients (TC and NI), and this is highlighted by the gray rectangle (top graph). Analysis of mean IgG reactivity (middle graph) was performed on three pairs of samples referred to as Chagas disease patients (CD vs. CDt), patients with the indeterminate clinical form of Chagas disease (IND vs. INDt) and patients with clinical manifestations of Chagas disease (CARD/DIG vs. CARD/DIGt), evaluated before and nine years after etiological treatment with benznidazole (middle graph). Additional analyses on individual samples at selected serum dilutions (1:80-1:640) were also carried out for comparisons between IND vs. INDt and CARD/DIG vs. CARD/ DIGt (bottom graph). Comparative analyses between the pairs of clinical groups were performed using the nonparametric Wilcoxon test and significant differences at $\mathrm{p}<0.05$ are highlighted with an asterisk.
\& Castro 2002) are still important for to determining whether a post-therapeutic cure has been achieved in Chagas disease cases.

The purpose of the present investigation was to contribute to this important point by evaluating the clinical, parasitological and serological characteristics of a group of $28 \mathrm{CD}$ who were treated with benznidazole during the chronic phase of the infection. Despite the low number of samples, the results demonstrate that, when all patients are considered together (CDt), the overall clinical evolution rate observed (2\%/year) is very similar to what was previously described for untreated CD (Viotti et al. 1994, 2006, Gallerano \& Sosa 2000, Fabbro et al. 2007), However, the data analysis indicates that INDt present a clinical evolution rate of only $0.5 \% / y e a r$. This is lower in comparison to what has been observed by other authors in epidemiological and clinical studies of the un-treated IND in Brazil, from which rates ranging from $2 \%$ to $4 \%$ / year were recorded (Prata 2001, Dias 2006). Moreover, the rate observed for INDt was lower than that measured for patients presenting previous clinical manifestations of the disease (CARD/DIGt $=7.4 \% /$ year $)$. These findings are also lower than what was observed by Montoya (1998), who demonstrated an incidence of 1.5\%/year (3 times higher than that observed in this study) for Chagas disease cardiopathy among untreated indeterminate patients in a 10-year follow-up study performed in Berilo. It is interesting to note that the only case among the INDt patients that evolved towards a symptomatic clinical form was from the oldest patient in this group (patient 27; age: 37 years). This corroborates previous observations from other authors, which stated that the impact of the aetiological treatment on late chronic infection is not as evident as the impact on younger patients (Gallerano \& Sosa 2000, Cançado 2002), although treatment could interrupt

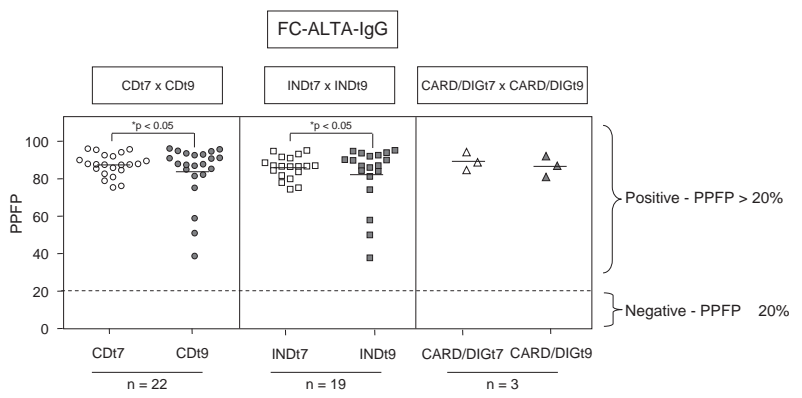

Fig. 5: serological reactivity of anti-Trypanosoma cruzi antibodies detected by non-conventional anti-live trypomastigote antibody analysis (FC-ALTA-IgG) in Chagas disease patients (CD), categorized as indeterminate (IND) or symptomatic (CARD/DIG) based on their clinical scores at the start of treatment. The data are reported as percentage of positive fluorescent parasites (PPFP), categorized as negative when PPFP $\leq 20 \%$ and positive when PPFP $>20 \%$, for individual serum samples collected seven years (CDt7, INDt7 and CARD/DIGt7) and nine years (CDt9, INDt9 and CARD/DIGt9) after etiological treatment with benznidazole. Comparative analyses of individual IgG reactivity between pairs of clinical groups were performed using the nonparametric Wilcoxon test and significant differences at $\mathrm{p}<0.05$ are highlighted with an asterisk. 
or delay patients' clinical evolution. Consequently, these data reinforce the importance of aetiological treatment for IND, as recommended by previous authors and by the Brazilian consensus resolution (Anonymous 2005) regarding specific treatment for Chagas disease.

The effect of the treatment on the chronic infection is controversial and difficult to demonstrate due to the limitation of the serological and parasitological methods available for cure detection. Several studies have demonstrated that treatment promotes remarkable reduction in the levels of antibodies and parasitaemia (Viotti et al. 1994, Fabro et al. 2007). Considering that the positive haemoculture rate in untreated patients in Berilo has been 36\% (unpublished observations), herein the results also demonstrate that the effect of treatment significantly reduced parasitaemia because the rate of positive blood culture in the treated group was only of $71.4 \%(2 / 28)$. Despite the low number of patients in this study, it is also interesting to note that positive results in treated patients were observed only within the CARD/DIGt group, which represents clear evidence of therapeutic failure. Parasitological tests (e.g., xenoculture and haemoculture) typically have very low sensitivity in the chronic phase of the disease and may yield negative results, even when repeated several times post-treatment (Castro et al. 2002). On the other hand, it has been demonstrated that PCR is the most sensitive method for detection of the parasite in the peripheral blood of patients in this phase of the infection (Britto et al. 2001).

Our results corroborated this observation, showing that PCR displays significantly higher sensitivity to demonstrate the presence of the parasite: only four out of the 28 patients $(14.8 \%)$ consistently displayed negative results and 23 (85.2\%) were positive when two different samples were evaluated. The first sample was $69.2 \%$ positive, while the second was $59.3 \%$ positive, yielding an agreement between the results of $57.7 \%$. This demonstrates the importance of the examination of at least two samples to increase the rate of parasite detection, as previously verified by Castro et al. (2002). Considering that patients in the chronic phase present low and intermittent parasitaemia, the parasites may not be detected in only one sample. Thus, negative PCR results should be interpreted with caution. Here, negative PCR results were observed exclusively in the INDt group. Although the real meaning of PCR results as a cure control has not been definitively determined (Martins et al. 2008, Britto 2009), the negative blood culture and PCR results observed in four INDt patients may be considered at least indicative of a significant reduction of parasitaemia. We did not observe a correlation between the negative parasitological tests and a reduction in the antibody levels, but patients who presented the greatest reduction of IgG also presented at a least one positive PCR reaction.

The serological evaluations using conventional ELISA and FC-ALTA-IgG demonstrate that all of the CDt patients presenting positive results may represent therapeutic failure, according to the cure criterion established by Krettly and Brener (1982) and MartinsFilho et al. (1995). With the aim of further focusing on the serological features of treated individuals, IgG reactivity was analysed using semiquantitative ELISA on serum samples from 16 paired samples, obtained before and nine years after treatment. The data analysis showed a significant decrease in antibody reactivity (particularly in INDt) in relation to IND, for all of the selected serum dilutions. It was demonstrated that the dilution of 1:320 is the most promising for discriminating the serological reactivities of anti-T. cruzi IgG antibodies. Three INDt (patients 1, 17 and 20) presented the highest decreases in IgG titers. No changes in OD values from semiquantitative ELISA were observed in the CARD/DIGt group. Interestingly, the patient who presented the largest decrease in IgG reactivity that was detected by ELISA (patient 1; age: 6 years) was the youngest patient treated. This represents an example of parasitological cure in progress, in accordance with the case mentioned by Luquetti \& Rassi (1998). This result also corroborates the observation that chemotherapy for Chagas disease is more successful in young people, especially under the age of 12 (Sosa-Estani \& Segura 2006). Interestingly, no significant changes in antibody levels were observed in patients within the CARD/ DIGt group. This result also corroborates affirmations regarding the real benefit from specific treatment for those in the chronic phase of Chagas disease (Ianni \& Mady 1998, Fabbro et al. 2006).

Although no CDt patients presented negative results in the FC-ALTA-IgG analysis (carried out 7 and 9 years after treatment), a significant decrease in PPFP values was observed in three patients, selectively in the INDt group (patients 1, 20 and 28). Two of these patients (1 and 20) were the same ones that presented decreased IgG reactivity in the semiquantitative ELISA test. No changes in PPFP values were observed in the CARD/DIGt patients.

When specific chemotherapy is administered to chronic patients, the decrease in serological titers of anti-T. cruzi antibodies occurs so slowly that the confirmation of cure assayed by conventional serology has only been achieved 10-25 years after treatment. These differences are due to the lower time of infection, methodological differences used in the evaluations and/or differences of the parasite's susceptibility to treatment of distinct geographical regions (Andrade et al. 1996, Coura \& Castro 2002). In fact, Galvão et al. (2003) have already demonstrated the early negativity of parasitological methods used among treated patients, in relation to serological methods. Therefore, we hypothesise that the presence of positive conventional ELISA, along with positive non-conventional FC-ALTA-IgG, that was observed in all CDt patients should not be considered therapeutic failure. Moreover, further evaluations should be carried out in such cases to fulfil the cure criteria proposed by Krettli and Brener (1982), Martins-Filho et al. (1995), Cançado (2002) and Coura and Castro (2002).

Taken together, after the cure criterion was adopted, no parasitological cure was observed in any of the treated CD patients whom we evaluated. However, considering the small number of samples, the data presented here suggest that even in the absence of cure, the treatment may have prevented or delayed the natural history 
of Chagas disease evolution in INDt, as demonstrated in other studies using considerable numbers of samples (Segura et al. 1994, Viotti et al. 1994, 2006, Galerrano \& Sosa 2000, Suasnábar et al. 2000, Streiger et al. 2004, Fabbro et al. 2007). Additionally, meta-analyses with relevant data about Chagas disease treatment have been previously reported by Villar et al. (2002), Reyes and Vallejo (2005) and Coura (2009).

Although this study was performed without evaluating a parallel standardised control group of untreated patients, our data suggest that benznidazole treatment produces beneficial effects selectively in the INDt group. This agrees with the view of the natural evolution of the disease described by Montoya (1998) in Berilo, who revealed that electrocardiographic alterations were present in $30.5 \%$ of CD patients, generally before 30 years old. This reality should be kept in mind by physicians when faced with newly diagnosed cases of Chagas disease.

The data demonstrate that the benefits for clinical evolution are selectively observed in the group of INDt patients and did not occur within the CARD/DIGt group. However, it is important to mention that the comparative analysis of clinical evolution rates with previously reported historical controls should be performed with caution since several variables (e.g., age, sex and exposition to infected vectors) may vary in different geographical regions. In this context, most of the historical controls used for comparative analysis in our study included patients from both sexes with ages ranging from 17-50 years. Moreover, in general, all historical data came from studies performed in geographical areas where the vectorial re-exposition has been reported to be analogous to the one observed in our study population. We would also like to emphasise that due to its retrospective nature, this study presents some limitations, including the low number of available samples and the lack of an intrinsic control group. Further studies including a larger number of patients are still needed to validate the beneficial impact of specific treatment in chronic Chagas disease.

\section{ACKNOWLEDGEMENTS}

To the municipality of Berilo, for all the facilities offered for developing this study.

\section{REFERENCES}

Andrade AL, Zicker F, de Oliveira RM, Almeida Silva S, Luquetti A, Travassos LR, Almeida IC, de Andrade SS, de Andrade JG, Martelli CM 1996. Randomised trial of efficacy of benznidazole in treatment of early Trypanosoma cruzi infection. Lancet 348: 1407-1413.

Andrade SG, Stocker-Guerret S, Pimentel AS, Grimaud JA 1991. Reversibility of cardiac fibrosis in mice chronically infected with Trypanosoma cruzi under specific chemotherapy. Mem Inst Oswaldo Cruz 86: 187-200.

Anonymous 2005. Consenso Brasileiro em Doença de Chagas. Rev Soc Bras Med Trop 38: 5-26.

Bahia MT, Guedes PMM, Fietto JLR, Lana M, Toledo MJO 2005. Current advance in human and experimental Chagas disease chemotherapy. In RM Mohan, Global research network (Org.) research advances in antimicrobial agents and chemoterapy, Kerala, p. 99-121.

Bern C, Montgomery SP, Herwaldt BL, Rassi A Jr, Marin-Neto JA, Dantas RO, Maguire JH, Acquatella H, Morillo C, Kirchhoff LV,
Gilman RH, Reyes PA, Salvatella R, Moore AC 2008. Evaluation and treatment of Chagas disease in the United States: a systematic review. JAMA 299: 1134-1135.

Braga MS, Lauria-Pires L, Arganaraz ER, Nascimento RJ, Teixeira AR 2000. Persistent infections in chronic Chagas' disease patients treated with anti-Trypanosoma cruzi nitroderivatives. Rev Inst Med Trop Sao Paulo 42: 157-161.

Britto C, Silveira C, Cardoso MA, Marques P, Luquetti A, Macedo V, Fernandes O 2001. Parasite persistence in treated chagasic patients revealed by xenodiagnosis and polimerase chain reaction. Mem Inst Oswaldo Cruz 96: 823-826.

Britto CC 2009. Usefulness of PCR-based assays to assess drug efficacy in Chagas disease chemotherapy: value and limitations. Mem Inst Oswaldo Cruz 104 (Suppl. 1): 122-135.

Cançado JR 1997. Terapêutica específica. In JCP Dias, JR Coura, Clínica e terapêutica da doença de Chagas. Uma abordagem prática para o clínico geral, Ed. Fiocruz, Rio de Janeiro, p. 323-351.

Cançado JR 2002. Long term evaluation of etiological treatment of Chagas disease with benznidazole. Rev Inst Med Trop Sao Paulo 44: 29-37.

Castro AM, Luquetti AO, Rass A 2002. Blood culture and polymerase chain reaction for the diagnosis of the chronic phase of human infection with Trypanosoma cruzi. Parasitol Res 88: 894-900.

Chiari E, Dias JCP, Lana M, Chiari CA 1989. Hemoculture for the parasitological diagnosis of humam cronic Chagas disease. Rev Soc Bras Med Trop 22: 19-23.

Cordeiro FD, Martins-Filho OA, da Costa Rocha MO, Adad SJ, Correa-Oliveira R, Romanha AJ 2001. Anti-Trypanosoma cruzi immunoglobulin G1 can be a useful tool for diagnosis and prognosis of human Chagas' disease. Clin Diag Labor Immunol 8: 112-118.

Coura JR 2009. Present situation and new strategies for Chagas disease chemotherapy: a proposal. Mem Inst Oswaldo Cruz 104: 549-554.

Coura JR, Abreu LL, Wilcox F, Petana W 1997. Estudo comparativo com emprego de benznidazole, nifurtimox e placebo na forma crônica da doença de Chagas em área de campo com transmissão interrompida. Rev Soc Bras Med Trop 30: 139-144.

Coura JR, Castro SL 2002. A critical review on Chagas Disease chemotherapy. Mem Inst Oswaldo Cruz 97: 3-24.

Dias JCP 2002. O controle da doença de Chagas no Brasil. In AC Silveira. O controle da doença de Chagas nos países do Cone Sul da América, Faculdade de Medicina de Triângulo Mineiro, Uberaba, p. 145-239.

Dias JCP 2006. The treatment of Chagas disease (South American trypanosomiasis). Ann Intern Med 144: 772-774.

Fabbro DL, Streiger ML, Arias ED, Bizai ML, del Barco M, Amicone NA 2007. Trypanocide treatment among adults with chronic Chagas disease living in Santa Fe city (Argentina) over a mean follow-up of 21 years: parasitological, serological and clinical evolution. Rev Soc Bras Med Trop 40: 1-10.

Gallerano RR, Sosa RR 2000. Interventional study in the natural evolution of Chagas disease. Evaluation of specific antiparasitic treatment. Retrospective-prospective study of antiparasitic therapy. Rev Fac Cien Med Univ Nac Cordoba 57: 135-162.

Galvão LMC, Chiari E, Macedo AM, Luquetti AO, Silva AS, Andrade ALS 2003. PCR assay for monitoring Trypanosoma cruzi parasitemia in childhood after specific chemotherapy. J Clin Microbiol 41: 5066-5070.

Gomes ML, Macedo AM, Vago AR, Pena SDJ, Galvão LMC, Chiari E 1998. Trypanosoma cruzi: Optimization of polymerase chain reaction for detection in human blood. Exp Parasitol 88: 28-33. 
Ianni BM, Mady C 1998. Therapeutics for the chronic form of Chagas' disease. Is the etiological treatment effective? Arq Bras Cardiol 70: 59-61.

Krettli AU, Brener Z 1982. Resistance against Trypanosoma cruzi associated to anti-living trypomastigote antibodies. $J$ Immunol 128: 2009-2012.

Lauria-Pires L, Braga M, Vexenat AC, Nitz N, Simoes-Barbosa A, Tinoco DL, Teixeira AR 2000. Progressive chronic Chagas heart disease ten years after treatment with anti-Trypanosoma cruzi nitroderivatives. Am J Trop Med Hyg 63: 111-118.

Luquetti A, Rassi A 1998. Tratamiento de la enfermedad de Chagas en fase crônica: Critérios de cura convencionales: xenodiagnóstico, hemocultivo y serologia. Rev Patol Trop 27: 37-51.

Macedo V, Silveira CA 1987. Perspectiva da terapêutica específica da doença de Chagas. Experiências na forma indeterminada. Rev Soc Bras Med Trop 20: M24-M26.

Marin-Neto JA, Rassi A Jr, Avezum A Jr, Mattos AC, Rassi A 2009. The BENEFIT trial: testing the hypothesis that trypanocidal therapy is beneficial for patients with chronic Chagas heart disease. Mem Inst Oswaldo Cruz 104: 319-324.

Martins HR, Figueiredo LM, Valamiel-Silva JC, Carneiro CM, Machado-Coelho GL, Vitelli-Avelar DM, Bahia MT, MartinsFilho OA, Macedo AM, Lana M 2008. Persistence of PCRpositive tissue in benznidazole-treated mice with negative blood parasitological and serological tests in dual infections with Trypanosoma cruzi stocks from different genotypes. J Antimicrob Chemother 61: 1319-1327.

Martins-Filho OA, Pereira ME, Carvalho JF, Cançado JR, Brener Z 1995. Flow cytometry, a new approach to detect anti-live trypomastigote antibodies and monitor the efficacy of specific treatment in human Chagas' disease. Clin Diag Lab Immunol 2: 569-573.

Montoya RA 1998. Morbidade da doença de Chagas no município de Berilo, Minas Gerais, Brasil. Estudos seccional e longitudinal da cardiopatia chagásica crônica (1987-1997), PhD Thesis, Instituto Oswaldo Cruz-Fiocruz, Rio de Janeiro, 153 pp.

OMS/OPAS - Organización Mundial de la Salud/Organización Panamericana de la Salud 1974. Aspectos clínicos de la enfermedad de Chagas. Informe de una reunión conjunta OMS/OPS de investigadores. Boll Ofic Sanit Panam 76: 141-158.
Prata A 2001. Clinical and epidemiological aspects of Chagas disease. Lancet Infect Dis 1: 92-100.

Resende JM, Lauar KL, Oliveira AR 1960. Aspectos clínicos e radiológicos da aperistalsis do esôfago. Rev Bras Gastroenterol 12: $247-262$

Reyes PA, Vallejo M 2005. Trypanocidal drugs for late stage, symptomatic Chagas disease (Trypanosoma cruzi infection). Cochrane Database Syst Rev 19: CD004102.

Segura MA, Mollina de Aspi E, Basombria MA 1994. Reversibility of muscle and heart lesions in chronic Trypanosoma cruzi infected mice after late tripanosomicidal treatment. Mem Inst Oswaldo Cruz 89: 213-216.

Sosa-Estani S, Segura EL 2006. Etiological treatment in patients infected by Trypanosoma cruzi: experiences in Argentina. Curr Opin Infect Dis 19: 583-587.

Streiger ML, Del Barco ML, Fabbro DL, Arias, ED, Amicone NA 2004. Longitudinal study and specific chemotherapy in children with chronic Chagas' disease, residing in a low endemicity area of Argentina. Rev Soc Bras Med Trop 37: 365-375.

Suasnábar SA, Arias E, Streiger M, Piacenza M, Ingaramo M, Del Barco M, Amicone N 2000. Evolutive behavior towards cardiomyopathy of treated (nirfurtimox or benznidazole) and untreated chronic chagasic patients. Rev Inst Med Trop Sao Paulo 42: 99-109.

Villar JC, Marin-Neto JA, Ebrahim S, Yusuf S 2002. Trypanocidal drugs for chronic asymptomatic Trypanosoma cruzi infection. Cochrane Database Syst Rev 1: CD003463.

Viotti R, Vigliano C, Armenti H, Segura E 1994. Treatment of chronic Chagas' disease with benznidazolee: clinical and serologic evolution of patients with long-term follow-up. Am Heart J 127: 151-162.

Viotti R, Vigliano C, Lococo B, Bertocchi G, Petti M, Alvarez MG, Postan M, Armenti A 2006. Long-term cardiac outcomes of treating chronic Chagas disease with benznidazole versus no treatment: a nonrandomized trial. Ann Intern Med 144: 724-734.

Voller A, Bidwell DE, Bartlett A 1976. Enzyme immunoassays in diagnostic medicine. Theory and practice. Bull WHO 53: 55-65.

WHO 2005. Report of the cientific working group on Chagas disease Chagas disease. Available from: http://www.who.int/tdr/diseases/ chagas/swg_chagas.htm. 